such single-cell map precision instantiated in the spatial arrangement of neural connections? For orientation tuning, at least, several lines of evidence suggest that an orientationspecific bias in the spatial arrangement of feed-forward axon arbors plays a significant role ${ }^{12,13}$. But the scale of precision in axonal arrays - that is, the degree of bias in the axon arbors that converge at a given point in the cortex-remains unclear. Moreover, it is not yet known how the broad and overlapping dendritic processes of cortical neurons sample from this array, as well as those supplied by other axonal populations, and how they do so in a fashion that yields such precision.

At a more fundamental level, the function served by cortical maps remains a puzzle—one that is deepened further by Okhi and colleagues' observations on the microarchitecture of orientation tuning in rat visual cortex. Despite the presence of neurons that are well tuned for orientation and/or direction of motion in rat visual cortex, there is no sign of the orderly arrangements that are so apparent in cat visual cortex: the orientation preferences of nearby cells are uncorrelated. The lack of maps in the rat is consistent with results from other studies in rodents and indicates that the orderly mapping of stimulus features is not a prerequisite for constructing tuned cortical responses ${ }^{14}$. As the authors suggest, orderly mapping might contribute to differences in tuning sharpness, but this seems a modest gain for such a major difference in cortical architecture. Perhaps the significance lies less in generating the mapped properties and more in how additional dimensions of the visual scene are encoded in the responses of individual neurons. Although they respond similarly to grating stimuli, adjacent neurons in cat area 18 are likely to differ in their response to a variety of other properties of the stimulus (for example, phase, contrast or temporal properties). Such diversity of functionally distinct cell types may be significantly reduced in rodent visual cortex, obviating advantages for connectivity or pooling that clustering of neurons according to similar properties may endow ${ }^{7,15}$.

Whatever the reason for such species differences, new approaches for simultaneously visualizing the properties of large numbers of individual neurons in functioning circuits promise to add appreciably to our understanding of the complexities of cortical architecture. Exploring how the world is mapped in the brain just got a lot more exciting.

1. Ohki, K., Chung, S., Ch'ng, Y.H., Kara, P. \& Reid, R.C Nature 433, 597-603 (2005).

2. Blasdel, G.G. \& Salama, G. Nature 321, 579-585 (1986).

3. Bonhoeffer, T. \& Grinvald, A. Nature 353, 429-431 (1991).

4. Weliky, M., Bosking, W.H. \& Fitzpatrick, D. Nature 379, 725-728 (1996).

5. Shmuel, A. \& Grinvald, A. J. Neurosci. 16, 6945-6964 (1996).

6. Swindale, N.V., Matsubara, J.A. \& Cynader, M.S J. Neurosci. 7, 1414-1427 (1987).

7. DeAngelis, G.C., Ghose, G.M., Ohzawa, I. \& Freeman, R.D. J. Neurosci. 19, 4046-4064 (1999).

8. Hubel, D.H. \& Wiesel, T.N. Proc. R. Soc. Lond. B 198 1-59 (1977).

9. Maldonado, P.E., Godecke, I., Gray, C.M. \& Bonhoeffer T. Science 276, 1551-1555 (1997).

10. Das, A. \& Gilbert, C.D. Nature 387, 594-598 (1997)

11. Bosking, W.H., Crowley, J.C. \& Fitzpatrick, D. Nat. Neurosci. 5, 874-882 (2002).

12. Chapman, B., Zahs, K.R. \& Stryker, M.P. J. Neurosci. 11, 1347-1358 (1991).

13. Mooser, F., Bosking, W.H. \& Fitzpatrick, D. Nat Neurosci. 7, 872-879 (2004).

14. Van Hooser, S.D., Heimel, J.A., Chung, S., Nelson, S.B. \& Toth, L.J. J. Neurosci. 25, 19-28 (2005)

15. Koulakov, A.A. \& Chklovskii, D.B. Neuron 29, 519 527 (2001)

\title{
Disentangling simple from complex cells
}

A long-standing view of receptive field mechanisms in primary visual cortex (V1) neurons has been challenged recently; a paper in this issue now provides strong evidence to suggest that the textbooks have it right. Hubel and Wiesel were the first to describe neurons in cat V1 with elongated receptive fields, which respond to stimuli such as bars in a particular orientation. In 1962, they also described classes of neurons with distinctive receptive field structures. Simplecell receptive fields are made up of nonoverlapping subregions; either bright or dark bars in the appropriate location excite a particular cell. For complex cells, these subregions are overlapping. Hubel and Wiesel hypothesized that simple-cell receptive fields are constructed from systematically arranged inputs from the lateral geniculate nucleus of the thalamus, whereas complex-cell receptive fields are constructed by combining inputs from multiple simple cells.

More recent work, however, has led to the suggestion that two distinct classes of neural circuitry are not necessary to account for the different properties of simple and complex cells. Such views have been supported by evidence that whereas simple and complex cells seem to form two classes when evaluated by their spiking behavior, the synaptic input responses of both cell types form a unimodal distribution. It has been proposed that spike threshold differences could lead to this apparent separation of the cell types by their spiking behavior (N. Priebe et al., Nat. Neurosci. 7, 1113-1122, 2004). Now, Martinez and colleagues (p. 372) combine intracellular recording (to characterize the spatial distribution and interaction of responses to bright and dark stimuli; red and blue regions in the figure) with anatomy (to locate neurons within different cortical layers). They found that
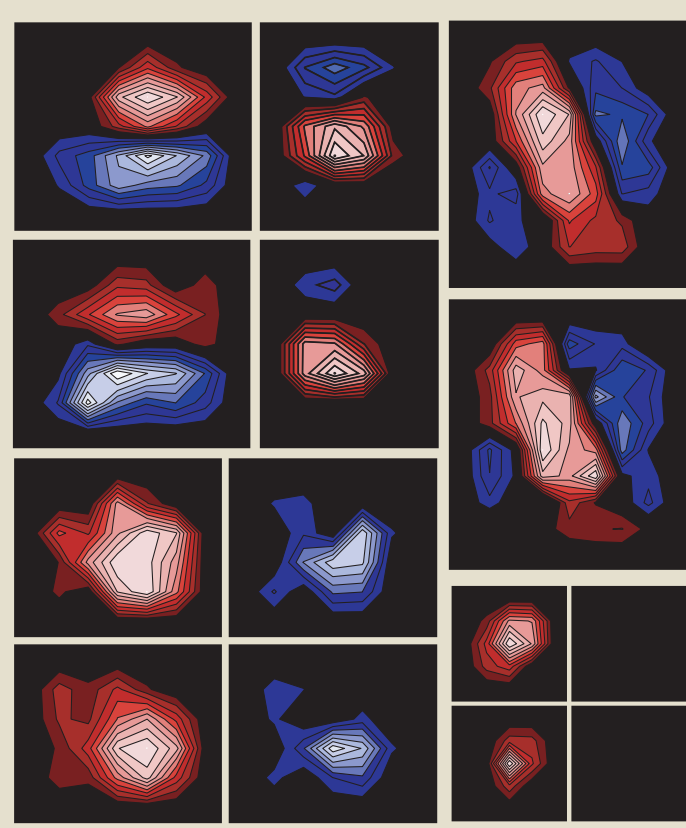
simple cells were exclusively located in layer 4 and upper layer
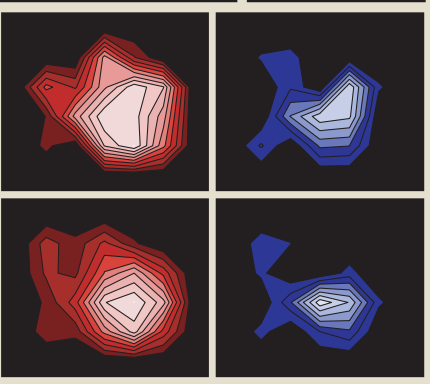
6 , the region where thalamic inputs enter the cortex. In contrast, complex cells were found in all layers, with cells in different layers having different receptive field structures. These results support the original proposal of Hubel and Wiesel that simple and complex cells belong to distinct neural circuits and that simple cells are an exclusive feature of an earlier stage of visual processing.

\section{I-han Chou}

